\title{
A Bicadmium-Substituted Polyoxometalate Network Based on a Vanadosilicate Cluster for the Selective Oxidation of Styrene to Benzaldehyde
}

Tong Zhou, Jie Zhang, Yuan-yuan Ma*, Xiaoru Gao, Qihui Xue, Yuanzhe Gao* and Zhan-gang Han*

Hebei Key Laboratory of Organic Functional Molecules, National Demonstration Center for Experimental Chemistry Education, College of Chemistry and Material Science, Hebei Normal University, Shijiazhuang, Hebei 050024, P. R. China

*Corresponding authors.*Email: mayy334@hebtu.edu.cn; yzgao2003@126.com, yzgao@hebtu.edu.cn; hanzg116@126.com,hanzg116@hebtu.edu.cn

\section{CONTENTS:}

Materials, methods, hydrothermal synthesis. $\mathrm{S} 2$

Table S1. Crystal data and structure refinement detail for $\mathbf{1} \quad$ S3

Table S2. The select bonds ( $\AA$ ) of compound $1 . \quad S 4$

Table S3. The select angles $\left(^{\circ}\right)$ of compound $1 . \quad$ S5

Table S4. The BVS calculations of metal elements in compound $1 . \quad$ S6

Figure $\mathrm{S} 1$. The $\left[(\mathrm{en})_{2} \mathrm{Cd}_{2} \mathrm{Si}_{8} \mathrm{~V}_{12} \mathrm{O}_{40}(\mathrm{OH})_{8}\right]^{4-}$ cluster act as a tetradentate ligand. $\mathrm{S} 6$

Figure S2. (a) and (b) The 2D network in compound 1. (c) Schematic view of the 3D supramolecular network of compound $\mathbf{1}$. S7

Figure S3. (a) IR spectra of compound 1; (b) Simulated and experimental powder XRD patterns of \begin{tabular}{lr} 
compound 1; (c) Thermogravimetric analysis of compound $\mathbf{1 .}$ & S8 \\
\hline
\end{tabular}

Figure S4. EDS data of compound 1, and the insert pictures are the SEM photos. $\quad$ S8

\begin{tabular}{lr} 
Figure S5. The recycle experiments with compound 1 as catalyst. & S9 \\
\hline
\end{tabular}

Figure S6. The IR spectra of compound 1 before and after the catalytic reaction. $\quad$ S9 


\section{Materials, methods and hydrothermal synthesis.}

All chemicals were used as purchased without purification. Hydrothermal synthesis was carried out with a $20 \mathrm{~mL}$ Teflon-lined autoclave under autogenous pressure, the reaction vessels were filled to approximately $25 \%$ of their volume capacity. IR spectra were recorded in $\mathrm{KBr}$ pellets with FTIR-8900 IR spectrometer in the range of 500-4000 $\mathrm{cm}^{-1}$. Powder X-ray diffraction (XRD) was performed on a Bruker AXS D8 Advance diffractometer. TG analyses were performed on a Perkin-Elmer Pyris Diamond TG/DTA instrument in following $\mathrm{N}_{2}$ with a heating rate of $10{ }^{\circ} \mathrm{C} \mathrm{min}^{-1}$.

Synthesis of 1: A mixture of $\mathrm{V}_{2} \mathrm{O}_{5}(0.092 \mathrm{~g}, 0.5 \mathrm{mmol}), \mathrm{Cd}\left(\mathrm{CH}_{3} \mathrm{COO}\right)_{2}(333 \mathrm{mg}$, $1.25 \mathrm{mmol})$, TEOS $(0.5 \mathrm{~mL})$, ethanol $(1 \mathrm{~mL})$, en $(0.35 \mathrm{~mL})$ and $\mathrm{H}_{2} \mathrm{O}(4 \mathrm{~mL})$ was

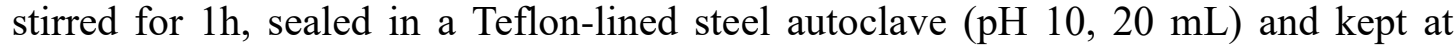
$170^{\circ} \mathrm{C}$ for 5 days. After the reactor was slowly cooled to room temperature, Dark block crystals of 1 were obtained, washed with distilled water, and air-dried to give a yield of $30 \%$ based on element $\mathrm{V}$.

The procedure for selective oxidative of styrene to benzaldehyde. In a typical case, $0.218 \mathrm{mmol}$ styrene and $0.020 \mathrm{mmol}$ catalyst were dispersed in $2 \mathrm{~mL} \mathrm{CH}_{3} \mathrm{CN}$ and stirred for $5 \mathrm{~min}$. Then, $30 \% \mathrm{H}_{2} \mathrm{O}_{2}(1 \mathrm{mmol})$ were injected to above admixture and heated at $70{ }^{\circ} \mathrm{C}$ for $4-12 \mathrm{~h}$. A small amount of the mixture was regularly removed and centrifuged. The filtrate was ascertained by GC analysis. After reaction, the catalyst was retrieved by centrifugation, washed with $\mathrm{CH}_{3} \mathrm{CN}, \mathrm{H}_{2} \mathrm{O}$ and $\mathrm{C}_{2} \mathrm{H}_{5} \mathrm{OH}$ alternately, and then dried in an oven at $70{ }^{\circ} \mathrm{C}$ for $24 \mathrm{~h}$. For the recyclability experiments, the reactions were conducted under the same reaction conditions. 


\section{Crystal analytical data.}

Single crystal data were collected on a Smart Apex CCD diffractometer at 296(2) $\mathrm{K}$ with Mo K $\alpha$ monochromated radiation $(\lambda=0.71073 \AA)$. The structures were solved by direct methods and refined by the full-matrix least-squares techniques on $F^{2}$ using the SHELXTL crystallographic software package ${ }^{[1,2]}$. Anisotropic thermal parameters were used to refine all nonhydrogen atoms. Hydrogen atoms attached to carbon atoms were added to their geometrically ideal positions and refined isotropically. Hydrogen atoms attached to water molecules were not identified from the difference Fourier map, but were added in the final formula. A summary of the crystallographic parameters of $\mathbf{1}$ is listed in Table S1. The selected bond distances $(\AA)$ and angles $\left(^{\circ}\right)$ for compound 1 are provided in Tables S2-S3

Table S1. Crystal data and structure refinement detail for $\mathbf{1}$.

\begin{tabular}{cc}
\hline Compound & 1 \\
\hline Empirical formula & $\mathrm{C}_{12} \mathrm{H}_{54} \mathrm{Cd}_{4} \mathrm{~N}_{12} \mathrm{O}_{53.50} \mathrm{Si}_{8} \mathrm{~V}_{12}$ \\
Formula weight & 2508.27 \\
Temperature $/ \mathrm{K}$ & 296.15 \\
Wavelength & $0.71073 \mathrm{~A}$ \\
Crystal system & Monoclinic \\
space group & $C 12 / c 1$ \\
$a / \AA$ & $19.9381(13)$ \\
$b / \AA$ & $14.8467(10)$ \\
$c / \AA$ & $23.5825(16)$ \\
$\alpha /{ }^{\circ}$ & 90 \\
$\beta /{ }^{\circ}$ & $102.2050(10)$ \\
$\gamma /{ }^{\circ}$ & 90 \\
Volume $/ \AA^{3}$ & $6823.0(8)$ \\
$\mathrm{Z}$ & 4 \\
Calculated density $\left(\mathrm{Mg} \mathrm{m}^{-3}\right)$ & 2.424 \\
Absorption coefficient $\left(\mathrm{mm}^{-1}\right)$ & 3.034 \\
$\theta$ for data collection $\left({ }^{\circ}\right)$ & 1.724 to 25.049 \\
$\mathrm{~F}(000)$ & 4872 \\
Reflections collected & 16925 \\
Reflections unique & 6028 \\
\hline int & 0.0158 \\
\hline
\end{tabular}




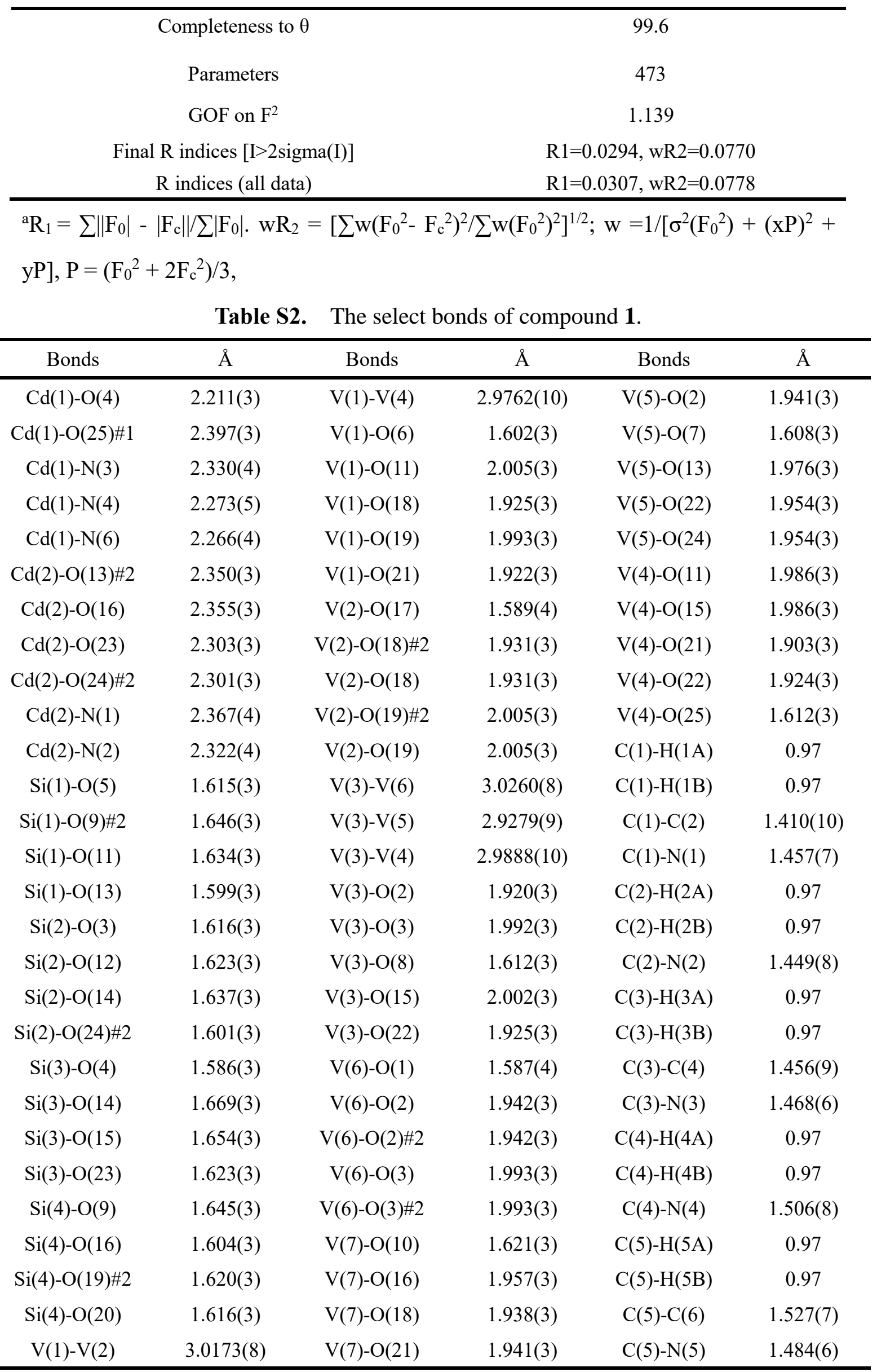




\begin{tabular}{llllll}
\hline $\mathrm{V}(1)-\mathrm{V}(7)$ & $2.9123(9)$ & $\mathrm{V}(7)-\mathrm{O}(23)$ & $1.969(3)$ & $\mathrm{C}(6)-\mathrm{H}(6 \mathrm{~A})$ & 0.97 \\
\hline
\end{tabular}

For 1: \#1 -x+1/2,-y+5/2,-z+1; \#2-x,y,-z+1/2

Table S3. The select angles of compound $\mathbf{1 .}$

\begin{tabular}{|c|c|c|c|}
\hline Angles & $\circ$ & Angles & $\circ$ \\
\hline $\mathrm{O}(4)-\mathrm{Cd}(1)-\mathrm{O}(25) \# 1$ & $87.63(10)$ & $\mathrm{O}(24) \# 2-\mathrm{Si}(2)-\mathrm{O}(14)$ & $108.75(15)$ \\
\hline $\mathrm{O}(4)-\mathrm{Cd}(1)-\mathrm{N}(3)$ & $93.41(13)$ & $\mathrm{O}(4)-\mathrm{Si}(3)-\mathrm{O}(14)$ & $107.79(15)$ \\
\hline $\mathrm{O}(4)-\mathrm{Cd}(1)-\mathrm{N}(4)$ & $115.30(19)$ & $\mathrm{O}(4)-\mathrm{Si}(3)-\mathrm{O}(15)$ & $112.64(15)$ \\
\hline $\mathrm{O}(4)-\mathrm{Cd}(1)-\mathrm{N}(6)$ & $104.94(13)$ & $\mathrm{O}(4)-\mathrm{Si}(3)-\mathrm{O}(23)$ & $115.59(16)$ \\
\hline $\mathrm{N}(3)-\mathrm{Cd}(1)-\mathrm{O}(25) \# 1$ & $82.31(12)$ & $\mathrm{O}(15)-\mathrm{Si}(3)-\mathrm{O}(14)$ & $102.79(14)$ \\
\hline $\mathrm{N}(4)-\mathrm{Cd}(1)-\mathrm{O}(25) \# 1$ & $149.54(17)$ & $\mathrm{O}(23)-\mathrm{Si}(3)-\mathrm{O}(14)$ & $105.76(14)$ \\
\hline $\mathrm{N}(4)-\mathrm{Cd}(1)-\mathrm{N}(3)$ & $76.83(16)$ & $\mathrm{O}(23)-\mathrm{Si}(3)-\mathrm{O}(15)$ & $111.14(15)$ \\
\hline $\mathrm{N}(6)-\mathrm{Cd}(1)-\mathrm{O}(25) \# 1$ & $83.74(13)$ & $\mathrm{O}(16)-\mathrm{Si}(4)-\mathrm{O}(9)$ & $107.61(15)$ \\
\hline $\mathrm{N}(6)-\mathrm{Cd}(1)-\mathrm{N}(3)$ & $156.40(15)$ & $\mathrm{O}(16)-\mathrm{Si}(4)-\mathrm{O}(19) \# 2$ & $113.35(15)$ \\
\hline $\mathrm{N}(6)-\mathrm{Cd}(1)-\mathrm{N}(4)$ & $107.41(17)$ & $\mathrm{O}(16)-\mathrm{Si}(4)-\mathrm{O}(20)$ & $115.06(16)$ \\
\hline $\mathrm{O}(13) \# 2-\mathrm{Cd}(2)-\mathrm{O}(16)$ & $85.92(10)$ & $\mathrm{O}(19) \# 2-\mathrm{Si}(4)-\mathrm{O}(9)$ & $107.11(14)$ \\
\hline $\mathrm{O}(13) \# 2-\mathrm{Cd}(2)-\mathrm{N}(1)$ & $117.06(14)$ & $\mathrm{O}(20)-\mathrm{Si}(4)-\mathrm{O}(9)$ & $107.26(15)$ \\
\hline $\mathrm{O}(16)-\mathrm{Cd}(2)-\mathrm{N}(1)$ & $151.44(14)$ & $\mathrm{O}(20)-\mathrm{Si}(4)-\mathrm{O}(19) \# 2$ & $106.03(16)$ \\
\hline $\mathrm{O}(23)-\mathrm{Cd}(2)-\mathrm{O}(13) \# 2$ & $125.67(10)$ & $\mathrm{V}(7)-\mathrm{V}(1)-\mathrm{V}(2)$ & $78.00(2)$ \\
\hline $\mathrm{O}(23)-\mathrm{Cd}(2)-\mathrm{O}(16)$ & $67.27(9)$ & $\mathrm{O}(18)-\mathrm{V}(1)-\mathrm{V}(4)$ & $114.90(9)$ \\
\hline $\mathrm{O}(23)-\mathrm{Cd}(2)-\mathrm{N}(1)$ & $106.61(13)$ & $\mathrm{O}(19)-\mathrm{V}(1)-\mathrm{O}(11)$ & $90.23(11)$ \\
\hline $\mathrm{O}(23)-\mathrm{Cd}(2)-\mathrm{N}(2)$ & $127.60(14)$ & $\mathrm{O}(21)-\mathrm{V}(1)-\mathrm{V}(2)$ & $114.03(8)$ \\
\hline $\mathrm{O}(24) \# 2-\mathrm{Cd}(2)-\mathrm{O}(13) \# 2$ & $66.95(10)$ & $\mathrm{O}(17)-\mathrm{V}(2)-\mathrm{O}(18)$ & $110.71(9)$ \\
\hline $\mathrm{O}(24) \# 2-\mathrm{Cd}(2)-\mathrm{O}(16)$ & $121.20(10)$ & $\mathrm{O}(17)-\mathrm{V}(2)-\mathrm{O}(18) \# 2$ & $110.71(9)$ \\
\hline $\mathrm{O}(24) \# 2-\mathrm{Cd}(2)-\mathrm{O}(23)$ & $87.28(10)$ & $\mathrm{O}(18) \# 2-\mathrm{V}(2)-\mathrm{O}(19)$ & $88.99(11)$ \\
\hline $\mathrm{O}(24) \# 2-\mathrm{Cd}(2)-\mathrm{N}(1)$ & $85.11(13)$ & $\mathrm{O}(19) \# 2-\mathrm{V}(2)-\mathrm{V}(1) \# 2$ & $40.84(8)$ \\
\hline $\mathrm{O}(24) \# 2-\mathrm{Cd}(2)-\mathrm{N}(2)$ & $143.06(13)$ & $\mathrm{O}(2)-\mathrm{V}(3)-\mathrm{O}(22)$ & $81.79(12)$ \\
\hline $\mathrm{N}(2)-\mathrm{Cd}(2)-\mathrm{O}(13) \# 2$ & $95.41(13)$ & $\mathrm{O}(3)-\mathrm{V}(3)-\mathrm{V}(6)$ & $40.61(8)$ \\
\hline $\mathrm{N}(2)-\mathrm{Cd}(2)-\mathrm{O}(16)$ & $87.59(12)$ & $\mathrm{V}(3)-\mathrm{V}(6)-\mathrm{V}(3) \# 2$ & $138.51(4)$ \\
\hline $\mathrm{N}(2)-\mathrm{Cd}(2)-\mathrm{N}(1)$ & $74.26(15)$ & $\mathrm{O}(2) \# 2-\mathrm{V}(6)-\mathrm{O}(3)$ & $90.61(11)$ \\
\hline $\mathrm{O}(5)-\mathrm{Si}(1)-\mathrm{O}(9) \# 2$ & $107.76(16)$ & $\mathrm{O}(2) \# 2-\mathrm{V}(6)-\mathrm{O}(3) \# 2$ & $78.58(11)$ \\
\hline $\mathrm{O}(5)-\mathrm{Si}(1)-\mathrm{O}(11)$ & $108.99(16)$ & $\mathrm{O}(10)-\mathrm{V}(7)-\mathrm{O}(23)$ & $105.90(14)$ \\
\hline $\mathrm{O}(11)-\mathrm{Si}(1)-\mathrm{O}(9) \# 2$ & $105.73(14)$ & $\mathrm{O}(16)-\mathrm{V}(7)-\mathrm{V}(1)$ & $125.48(9)$ \\
\hline $\mathrm{O}(13)-\mathrm{Si}(1)-\mathrm{O}(5)$ & $113.16(17)$ & $\mathrm{O}(16)-\mathrm{V}(7)-\mathrm{O}(23)$ & $82.17(12)$ \\
\hline $\mathrm{O}(13)-\mathrm{Si}(1)-\mathrm{O}(9) \# 2$ & $108.74(15)$ & $\mathrm{O}(18)-\mathrm{V}(7)-\mathrm{V}(1)$ & $40.90(8)$ \\
\hline $\mathrm{O}(13)-\mathrm{Si}(1)-\mathrm{O}(11)$ & $112.10(16)$ & $\mathrm{Si}(1)-\mathrm{O}(13)-\mathrm{V}(5)$ & $130.20(17)$ \\
\hline $\mathrm{O}(3)-\mathrm{Si}(2)-\mathrm{O}(12)$ & $107.38(16)$ & $\mathrm{V}(5)-\mathrm{O}(13)-\mathrm{Cd}(2) \# 2$ & $104.21(12)$ \\
\hline $\mathrm{O}(3)-\mathrm{Si}(2)-\mathrm{O}(14)$ & $106.15(15)$ & $\mathrm{Si}(2)-\mathrm{O}(14)-\mathrm{Si}(3)$ & $125.67(17)$ \\
\hline $\mathrm{O}(12)-\mathrm{Si}(2)-\mathrm{O}(14)$ & $107.90(15)$ & $\mathrm{C}(1)-\mathrm{C}(2)-\mathrm{N}(2)$ & $115.3(6)$ \\
\hline $\mathrm{O}(24) \# 2-\mathrm{Si}(2)-\mathrm{O}(3)$ & $113.36(16)$ & $\mathrm{H}(2 \mathrm{~A})-\mathrm{C}(2)-\mathrm{H}(2 \mathrm{~B})$ & 107.5 \\
\hline $\mathrm{O}(24) \# 2-\mathrm{Si}(2)-\mathrm{O}(12)$ & $112.97(17)$ & $\mathrm{N}(2)-\mathrm{C}(2)-\mathrm{H}(2 \mathrm{~A})$ & 108.5 \\
\hline
\end{tabular}

For 1: $\# 1-\mathrm{x}+1 / 2,-\mathrm{y}+5 / 2,-\mathrm{z}+1 ;$ \#2-x,y,-z+1/2 
Table S4. The BVS calculations of metal elements in compound 1

\begin{tabular}{llll}
\hline elements & valence state & elements & valence state \\
\hline Si1 & 4.010 & V3 & 4.086 \\
Si2 & 4.054 & V4 & 4.158 \\
Si3 & 3.921 & V5 & 4.123 \\
Si4 & 4.033 & V6 & 4.149 \\
V1 & 4.128 & V7 & 4.105 \\
V2 & 4.139 & & \\
\hline
\end{tabular}

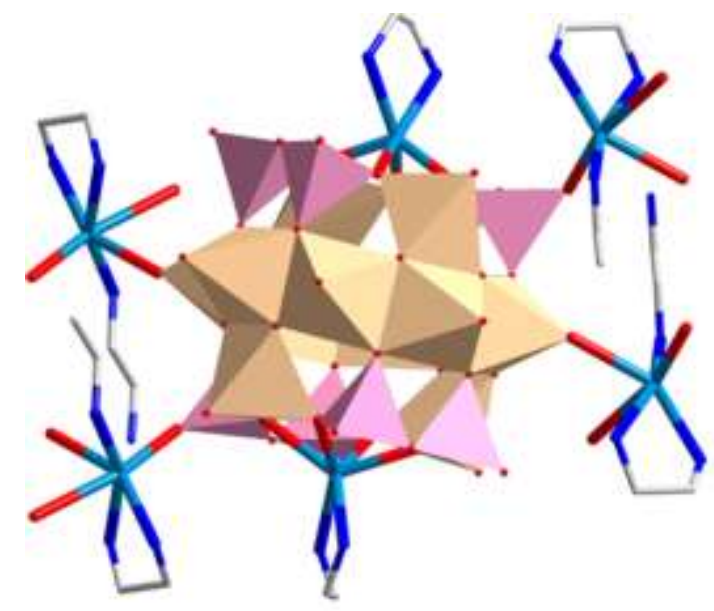

Figure $\mathrm{S1}$. The $\left[(\mathrm{en})_{2} \mathrm{Cd}_{2} \mathrm{Si}_{8} \mathrm{~V}_{12} \mathrm{O}_{40}(\mathrm{OH})_{8}\left(\mathrm{H}_{2} \mathrm{O}\right)_{0.5}\right]^{4-}$ cluster act as a tetradentate ligand. 
(a)

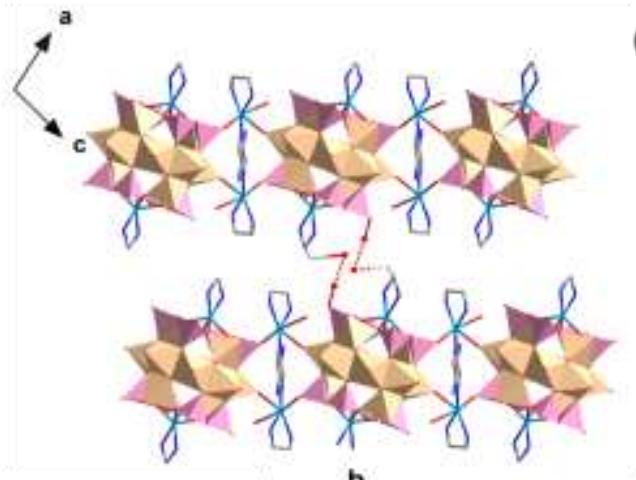

(c)

b (b)

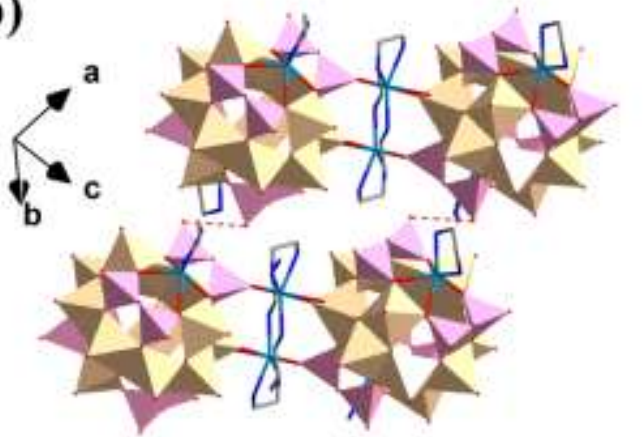

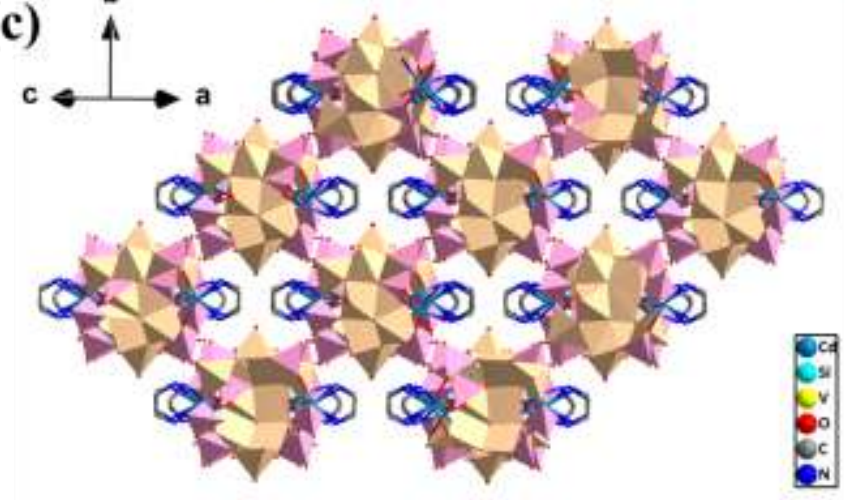

Figure S2. (a) and (b) The 2D network in compound 1. (c) Schematic view of the 3D supramolecular network of compound $\mathbf{1}$.

\section{IR, XRD, TG and EDS analysis.}

In addition, the physical and chemical properties of compound $\mathbf{1}$ were studied by IR spectra, XRD patterns and thermogravimetric analysis. As shown in Figure S3a, the IR spectra show that the strong peaks at $1116-997 \mathrm{~cm}^{-1}$ can be attributed to the stretching vibrations of $\mathrm{V}=\mathrm{O}$ bonds, whereas the strong peaks at $877-616 \mathrm{~cm}^{-1}$ may be due to the $\mathrm{Si}-\mathrm{O}$ stretching vibration of the $\left\{\mathrm{Si}_{2} \mathrm{O}_{7}\right\}$ dimer. Moreover, the strong absorption peaks in the range of $1644-1460 \mathrm{~cm}^{-1}$ are attributed to the vibrations of $\mathrm{C}=\mathrm{C}$ and $\mathrm{C}-\mathrm{N}$ bonds in organic ligand en. The broad band near $3400 \mathrm{~cm}^{-1}$ show the intermolecular hydrogen bonding interaction ${ }^{[3]}$. Figure S3b shows the powder XRD patterns of compound 1. A good accordance between the experimental and simulated powder XRD patterns has been observed, indicating the good phase purity of the samples. The differences in reflection intensity are probably due to preferential orientations in the powder samples of $\mathbf{1}$. Thermogravimetric analysis was carried out in the temperature range of $25-800{ }^{\circ} \mathrm{C}$ under an atmosphere of $\mathrm{N}_{2}$ (Figure S3c), confirming the good stability of compound $\mathbf{1}$ below $286^{\circ} \mathrm{C}$. In order to further prove 
the ratios of $\mathrm{Cd}, \mathrm{Si}$ and $\mathrm{V}$ in $\mathbf{1}$, the content of element was analyzed by using scanning electron microscope at energy spectrometer. The results are shown in Figure S4, illustrating that the ratios of $\mathrm{Cd}, \mathrm{Si}$ and $\mathrm{V}$ atoms in compound 1 are $1: 2: 3$.
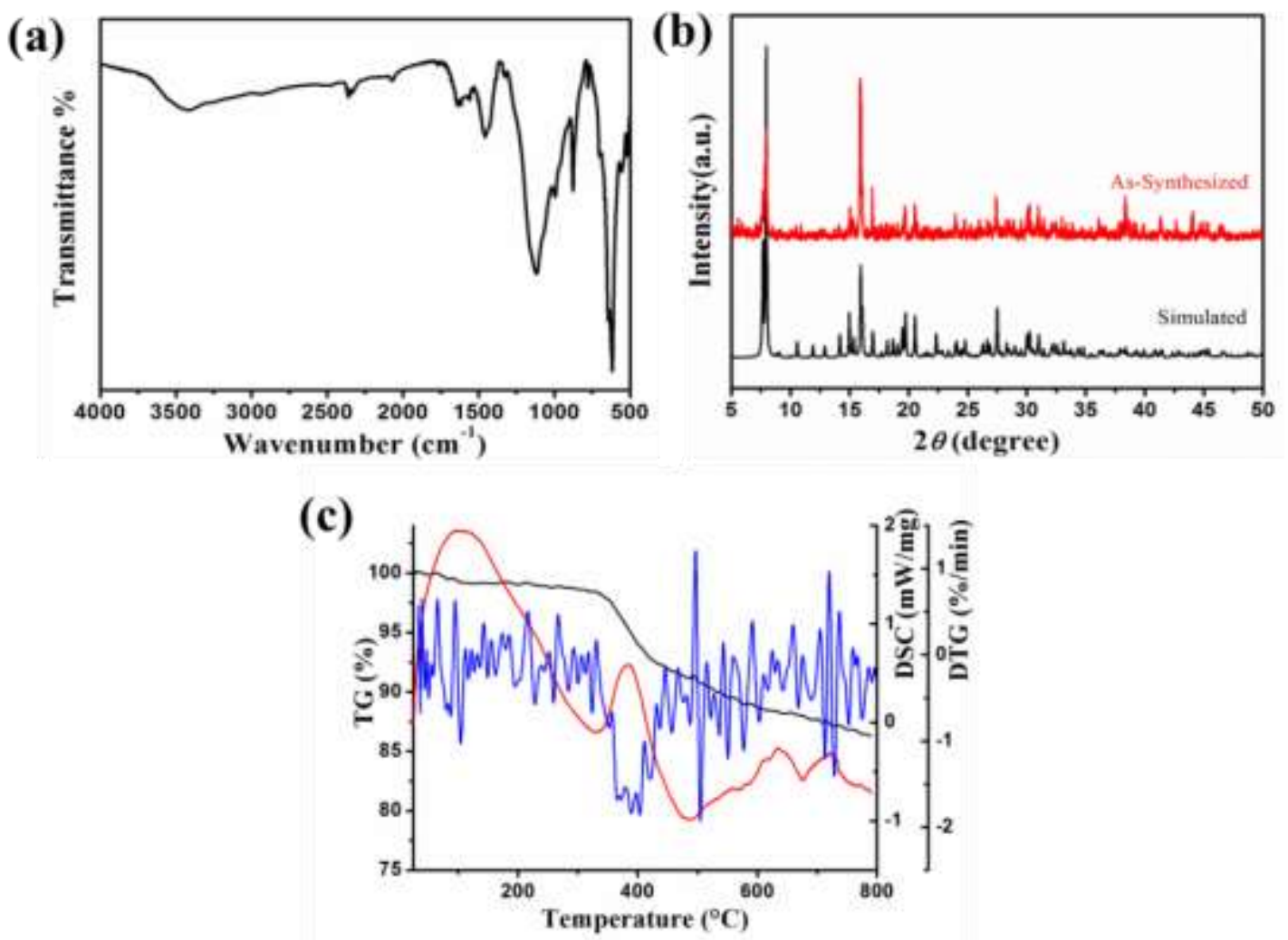

Figure S3. (a) IR spectra of compound 1. (b) Simulated and experimental powder XRD patterns of compound 1. (c) Thermogravimetric analysis of compound $\mathbf{1}$.

\begin{tabular}{ccc}
\hline \multirow{2}{*}{ element } & \multicolumn{2}{c}{ compound $\mathbf{1}$} \\
\cline { 2 - 3 } & mass \% & atom \% \\
\hline $\mathrm{C}$ & 7.09 & 13.36 \\
$\mathrm{~N}$ & 9.09 & 14.68 \\
$\mathrm{O}$ & 36.92 & 52.22 \\
$\mathrm{Si}$ & 9.14 & 7.37 \\
$\mathrm{~V}$ & 19.63 & 8.72 \\
$\mathrm{Cd}$ & 18.12 & 3.65 \\
gross & 100.0 & \\
\hline
\end{tabular}

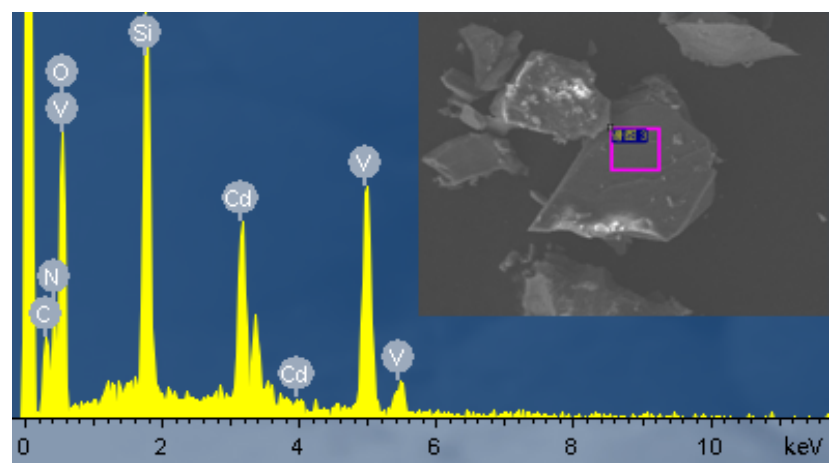

Figure S4. EDS data of compound 1, and the insert pictures are the SEM photos. 


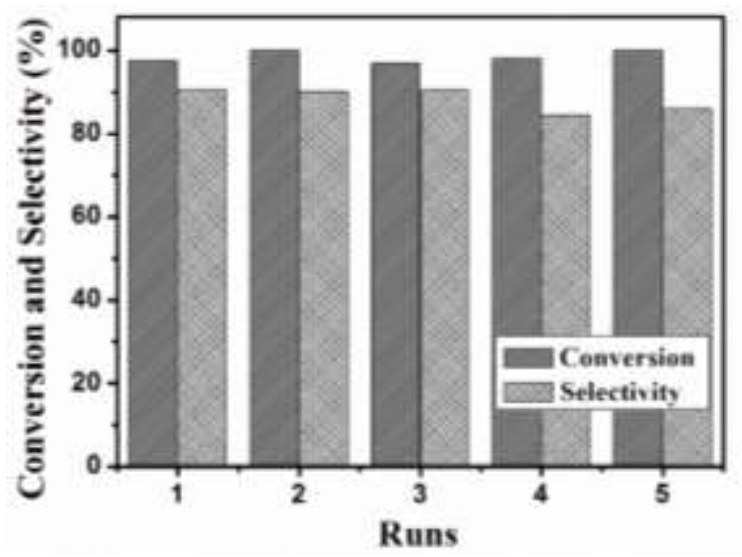

Figure S5. The recycle experiments with compound $\mathbf{1}$ as catalyst.

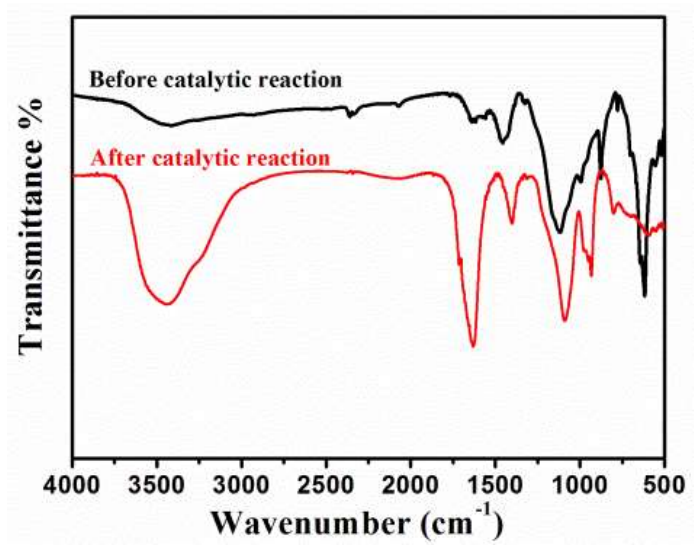

Figure S6. The IR spectra of compound $\mathbf{1}$ before and after the catalytic reaction.

\section{Reference}

(1) Sheldrick, G. A short history of SHELXL. Acta Cryst. 2008, A64, 112-122.

(2) Sheldrick, G. Crystal structure refinement with SHELXL. Acta Cryst. 2015, C71, 3-8.

(3) Li, N.; He, D. F.; Lu, Y.; Liu, Y. W.; Xu, L.; Zhong, X. H.; Guo, Z. H.; Liu, S. X. A Vanadogermanate Dimer-Based Chain with Magnetic and Luminescent Properties. Eur. J. Inorg. Chem. 2016, 3143-3147. 